\title{
CryoEM map of Pseudomonas aeruginosa PilQ enables structural
}

\section{characterization of TsaP}

3 Matthew McCallum ${ }^{1,2, \dagger, *}$, Stephanie Tammam ${ }^{1, \dagger}$, John L. Rubinstein ${ }^{1,2,3, *}$, Lori L. Burrows ${ }^{4, *}$, P.

$6 \quad{ }^{1}$ Program in Molecular Structure \& Function, Peter Gilgan Centre for Research and Learning, The Hospital for Sick Children, Toronto, ON M5G 0A4, Canada.

$10{ }^{4}$ Department of Biochemistry and Biomedical Sciences and the Michael G. DeGroote Institute for 11 Infectious Disease Research, McMaster University, Hamilton, ON L8S 4K1, Canada.

13 "To whom correspondence should be addressed:

14 howell@sickkids.ca, lori.burrows@mcmaster.ca, john.rubinstein@utoronto.ca

16 Lead contact: PLH

17

18 These authors contributed equally to this work

19 Current address: Department of Biochemistry, University of Washington, Seattle, WA 98195207350, USA. 


\section{ABSTRACT}

The type IV pilus machinery is a multi-protein complex that polymerizes and

23 depolymerizes a pilus fibre used for attachment, twitching motility, phage adsorption, natural

24 competence, protein secretion, and surface-sensing. An outer membrane secretin pore is required

25 for passage of the pilus fibre out of the cell. Herein, the structure of the tetradecameric secretin,

26 PilQ, from the Pseudomonas aeruginosa type IVa pilus system was determined to $4.3 \AA$ and 4.4

$27 \AA$ resolution in the presence and absence of $\mathrm{C}_{7}$ symmetric spokes, respectively. The heptameric

28 spokes were found to be the two tandem C-terminal domains of TsaP. TsaP forms a belt around

29 PilQ and while the protein is not essential for twitching motility, over-expression of TsaP triggers

30 a signal cascade upstream of PilY1 leading to cyclic di-GMP up-regulation. These results resolve

31 the identity of the spokes identified with Proteobacterial PilQ homologs and may reveal a new

32 component of the surface-sensing cyclic di-GMP signal cascade.

\section{IMPACT STATEMENT}

35 The type IV pilus is critical for bacterial virulence. The co-structure of the pilus secretin PilQ and

36 TsaP is determined. Characterization of TsaP implicates it in surface-sensing signal transduction.

\section{INTRODUCTION}

39 The type IVa pilus (T4aP) facilitates many bacterial functions including attachment,

40 twitching motility, phage adsorption, natural competence, protein secretion, and surface-

41 sensing ${ }^{1,2}$. The surface-sensing role of $\mathrm{T} 4 \mathrm{aP}$ is of particular interest due to its involvement in the

42 up-regulation of cyclic di-GMP - the master regulator of biofilm formation ${ }^{3,4}$. Biofilms are of

43 critical medical and industrial importance, as they increase bacterial tolerance of otherwise

44 uninhabitable conditions, antibiotics, and to the immune system ${ }^{5}$.

45 The T4aP machinery comprises a cell envelope-spanning multi-protein complex. This

46 machinery polymerizes long thin fibres that attach to surfaces and retract to pull bacteria towards

47 the point of attachment ${ }^{1,2}$. The pilus is a polymer mainly composed of the major subunit, PilA. At

48 the pilus base, the inner membrane protein PilC and cytoplasmic ATPase motors, PilB and PilT,

49 catalyze polymerization and depolymerization, respectively ${ }^{6,7}$. Minor pilins and PilY1 initiate

50 pilus polymerization and are thus thought to localize at the pilus tip ${ }^{8,9}$. The pilus exits the

51 periplasm through an outer membrane pore formed by PilQ oligomers. The PilQ pore is aligned 
52 with the motors by a subcomplex of PilM, PilN, PilO, and PilP ${ }^{10,11}$. PilF shuttles PilQ to the outer

53 membrane and facilitates its oligomerization. PilMNOP, PilY1, and PilT are all required for

$54 \mathrm{~T} 4 \mathrm{aP}-$ mediated surface sensing ${ }^{12,13}$. It is unclear whether other proteins that are part of the T4aP

55 apparatus and interface with PilM, PilN, PilO, and PilP - such as PilQ - are also needed for

56 surface sensing.

PilQ and PilF are the secretin and pilotin of the T4aP, respectively. Secretins and their cognate pilotins create outer-membrane channels in various secretion and pilus systems. Secretin

59 oligomers have a unique double-layered $\beta$-barrel architecture ${ }^{14,15}$. The outer $\beta$-barrel forms a

60 large vestibule that passes though the outer membrane ${ }^{15}$. The inner $\beta$-barrel curves inwards to

61 form a gate at the midway point of the secretin $\beta$-barre ${ }^{15}$. Domains N1 and N0 form rings below

62 the $\beta$-barrel ${ }^{15}$. PilQ secretins from Proteobacteria, structurally characterized at low resolution,

63 show radial spokes emerging from a central barrel ${ }^{16-19}$. Models of PilQ from Thermus

64 thermophilus $\left(\mathrm{PilQ}^{\mathrm{Tt}}\right)$ and Vibrio cholerae $\left(\mathrm{PilQ}^{\mathrm{Vc}}\right)$ competence pilus systems - which are

65 divergent from Proteobacterial T4aP secretins - were recently built into 7 and $2.7 \AA$ resolution

66 electron cryomicroscopy (cryoEM) maps, respectively ${ }^{20,21}$. These competence secretins lack the

67 lip subdomain and the radial spokes found in $\mathrm{T} 4 \mathrm{aP}$ secretins.

68 Structures of the related type II secretion system (T2SS) secretins also have radial

69 spokes $^{22,23}$. These structures were determined at 3-4 $\AA$ resolution, permitting modeling of the

70 spoke protein as the T2SS pilotin ${ }^{22,23}$. This finding suggests that the spokes in the T4aP structures

71 could be the T4aP pilotin, PilF. However, PilF is structurally unrelated to the T2SS pilotin, and

72 other studies indicate that the spokes are most likely the protein $\mathrm{TsaP}^{16,19}$. 2D class averages of

73 cryoEM particles embedded in purified Neisseria sp. membranes show spokes around the PilQ

74 barrel, and these spokes disappear upon deletion of $\mathrm{TsaP}^{16}$. Similarly, electron cryotomography

75 of Myxococcus xanthus show that density consistent with the location of the spokes around PilQ

76 disappears upon deletion of $\mathrm{TsaP}^{19}$. Little is known about the molecular function of TsaP, except

77 that it has a LysM domain characteristic of septal peptidoglycan binding ${ }^{16}$. Neither TsaP nor PilF

78 are not expressed in the same operon as PilQ.

79 We previously resolved a $7.4 \AA$ map of PilQ from Pseudomonas aeruginosa $\left(\mathrm{PilQ}{ }^{\mathrm{Pa}}\right.$ ) with

80 presumed tetradecameric stoichiometry and $C_{7}$ symmetric spoke density ${ }^{18}$. Although this was the

81 highest resolution structure of a secretin at that time, it was not sufficient for model building.

82 Herein, our reprocessing of those micrographs with improved image analysis and model-building 
83 algorithms yielded three distinct structures of $\mathrm{PilQ}^{\mathrm{Pa}}$. These maps were of sufficient resolution to

84 enable building of an atomic model. Mass spectrometry revealed the identity of the protein bound

85 in the spoke density to be TsaP, and a TsaP model was readily built into the spoke density. While

86 probing for the function of TsaP, we found that that its overexpression triggers cyclic di-GMP

87 up-regulation upstream of PilY1, implicating a role in the T4aP-dependent surface-sensing signal

88 cascade.

89

RESULTS

\section{$91 \quad$ PilQ $^{\mathrm{Pa}}$ forms tetradecameric and pentadecameric barrels}

We previously resolved a $7.4 \AA$ resolution $\mathrm{PilQ}^{\mathrm{Pa}}$ cryoEM map ${ }^{18}$. Using the template-

93 based automatic particle selection algorithm implemented in cryoSPARC v. $2^{24}$, eight times more

94 particles were selected automatically from the original micrographs than when particle selection

95 was performed manually (Supplementary Table 1). Ab initio classification followed by

96 heterogeneous particle refinement in 3D revealed three unique classes, with $44 \%$ of particle

97 images classified as $C_{14}$ symmetric barrels with $C_{7}$ symmetric radial spokes (Figure 1A), $48 \%$ of

98 particle images classifying as $C_{14}$ symmetric barrels without spokes (Figure 1B), and $8 \%$ as $C_{15}$

99 symmetric barrels without spokes (Figure 1C). Refining the three classes yielded maps at 4.3,

1004.4 , and $6.9 \AA$ resolution, respectively. The distinct $C_{14}$ and $C_{15}$ symmetric maps identified are

101 reminiscent of the finding that the T2SS secretin can have more than one symmetry ${ }^{25}$.

102 Local resolution in the barrel of the 4.3 and $4.4 \AA$ maps is estimated to be $3.5 \AA$. The

103 secondary structure and large side chains were visible in the map and enabled model building of

$10414 \mathrm{PilQ}^{\mathrm{Pa}} \mathrm{N} 1$ and secretin domains (Figure 1A). Although we verified that the N0 and

105 peptidoglycan-binding AMI_N domains of PilQ were present using mass spectrometry analyses

106 (Supplementary Figure 1), they could not be modeled, consistent with these domains adopting

107 random orientations relative to the secretin barrel in the absence of the peptidoglycan and the rest

108 of the T4aP apparatus. Protomers of the barrel structure from the spoked barrel map were fit into

109 the maps of the $C_{14}$ and $C_{15}$ barrels without spokes and refined.

110 The final models were compared to co-evolved residues within $\mathrm{PilQ}^{\mathrm{Pa}}$ homologs as

111 identified by the EVcouplings webserver ${ }^{26}$. Residues in proximity to one another in a structure

112 develop complementary mutations as the protein evolves ${ }^{27}$. These co-evolved residues can be

113 used to independently predict and validate the conformations of proteins ${ }^{27}$. We found a clear 
114 correlation between the co-evolved residues and the N1 and secretin domains, validating these

115 structures (Figure 1D). The sequence of the lip subdomain is not well conserved in PilQ, and the

116 poor sequence alignment in this region yielded fewer co-evolved residues in the lip subdomain

117 (Figure 1D).

118

119 Co-evolved residues are consistent with secretin gate and barrel dynamics

120 Co-evolved residues not directly supported by the model of $\mathrm{PilQ}^{\mathrm{Pa}}$ were also identified, 121 suggesting dynamics not visualized in the cryoEM maps. There are co-evolved residues between

122 the secretin gate and the secretin barrel (Figure 1E), consistent with a swinging gate-like model

123 of secretin gate opening demonstrated recently for the secretin from the type III secretion

124 injectisome, $\mathrm{InvG}^{28}$. Residue G577 is located at the proposed hinge of the P. aeruginosa PilQ

125 secretin gate (Figure 1E). Mutation of the corresponding residue in M. xanthus PilQ (G764S)

126 causes vancomycin sensitivity ${ }^{29}$ presumably because the hinge of the gate is jammed open

127 permitting entry of the bulky antibiotic across the outer membrane. This hypothesis is supported

128 by the structure of a mutant of the T2SS secretin where the corresponding residue G458 was

129 changed to alanine $(\mathrm{G} 458 \mathrm{~A})^{25}$. The G458A T2SS secretin structure reveals a partially open

130 secretin gate ${ }^{25}$.

131 Co-evolved residues were also identified at the top of the secretin barrel and near the

132 bottom of the secretin barrel, adjacent to the secretin gate (Figure 1F). These co-evolved residues

133 may be consistent with the secretin barrel folding such that the top of the secretin barrel covers

134 the gate-adjacent segment of the secretin. The top of the secretin is hydrophobic (Figure 1G),

135 like other secretin structures ${ }^{15}$, consistent with this region being the site for outer membrane

136 insertion. Thus, one explanation for these co-evolved residues may be that a folding intermediate

137 positions the top of the secretin barrel to mask these hydrophobic sites prior to membrane

138 insertion (Figure 1F). The gate-adjacent section of the secretin barrel is highly conserved

139 (Figure 1G) and provides the interface for the spoke density. Once PilQ inserts into the outer

140 membrane and this region becomes exposed, the spoke protein could bind and stabilize the PilQ

141 oligomer.

142

\section{Dynamics in the secretin gates visualized}


The conformations of the secretin gates in the $C_{15}, C_{14}$, and spoked $C_{14}$ PilQ ${ }^{\mathrm{Pa}}$ barrels are

145 different. While the secretin gate in the $C_{15}$ barrels is perpendicular to the walls of the barrel, the

146 secretin gate in the $C_{14}$ barrel invaginates towards the periplasm. Comparing the $C_{15}$ and $C_{14}$

147 barrels without spokes, the residues at the center of the secretin gate are displaced by $4 \AA$ (Figure

148 1B-C, Animation 1). This invagination is likely the result of the $5 \AA$ reduction in diameter of the

149 tetradecameric barrel relative to the pentadecameric form, leaving less room for the gate to

150 extend perpendicularly from the walls of the barrel.

Comparing the $C_{14}$ and spoked $C_{14}$ barrels reveals that the secretin gate invaginates by an

152 additional $3 \AA$ in the spoked $C_{14}$ barrel (Figure 1A-B). A smaller barrel diameter cannot explain

153 this movement, as the diameters of the $C_{14}$ barrel and spoked $C_{14}$ barrels are identical. Comparing

154 the $C_{15}, C_{14}$, and spoked $C_{14}$ barrels reveals a pivot point for gate invagination in the secretin

155 barrel adjacent to the secretin gate and the spokes (Figure 1H-I and Animation 1). This finding

156 is consistent with the molecules bound at the spokes promoting secretin gate invagination.

\section{TsaP co-purifies with PilQ}

159 There are two features in the maps of $\mathrm{PilQ}^{\mathrm{Pa}}$ that cannot be explained by models of PilQ ${ }^{\mathrm{Pa}}$

160 alone. First are the $C_{7}$ symmetric spokes. The spoke density appears to be comprised of 14

161 structurally similar $\beta$-roll protein structures that form a belt around PilQ. There are differences in

162 every other $\beta$-roll that give the spokes $C_{7}$ symmetry (Figure 1A). There is also extra density

163 beneath the secretin gate that plugs the small opening in the center of both tetradecameric PilQ

164 barrels - the density is more pronounced in the spoked tetradecameric barrel map (Figure 1A-B).

165 The local quality of the map beneath the secretin gate is too poor to identify distinct structural

166 elements. Curiously, there is no evidence of $C_{15}$ barrels with spokes or extra density beneath the

167 secretin gates, hinting at stoichiometry-dependent interactions.

168 To identify the protein(s) that constitute the extra densities, mass spectrometry was

169 performed on the protein sample used for cryoEM grid preparation. Aside from PilQ ${ }^{\mathrm{Pa}}$, the most

170 abundant components are PrpL, PA5122, and TsaP (Supplementary Table 2, Supplementary

171 Figure 2). TsaP is the protein most likely responsible for the spoke density as it has been shown

172 to be critical for PilQ spoke visualization in Neisseria sp. and M. xanthus ${ }^{16,19}$. PrpL is an

173 extracellular protease secreted by the $\mathrm{T}_{2} \mathrm{SS}^{30,31}$ and thus unlikely to constitute these densities.

174 PA5122 is an uncharacterized protein with a secretion signal sequence. 


\section{Model building of TsaP reveals unique heptamer structure}

The structure of TsaP is not known; however, TsaP was predicted to have an N-terminal

178 peptidoglycan-binding LysM domain linked by unstructured residues to a C-terminal domain ${ }^{19}$.

179 The C-terminal domain of TsaP, henceforth referred to as the CII domain, was predicted to be

180 similar to the $\mathrm{C}$-domain of the flagellar ring-forming component, $\mathrm{FlgT}^{19}$. The C-domain of FlgT

181 has a $\beta$-roll structure with an extruding finger subdomain ${ }^{32}$ and the Phyre $^{2}$ structure prediction of

182 TsaP from $P$. aeruginosa is consistent with this fold (Figure 2A). To validate this result, TsaP

183 co-evolved residues identified by the EVcouplings webserver ${ }^{26}$ were compared with the predicted

184 structure of the TsaP CII domain (Figure 2B). The correlations between co-evolved residues and

185 residues close to one another in the CII domain structure prediction provide support for its

186 similarity to the FlgT C-domain. Inspection of TsaP co-evolved residues revealed a duplicate

187 pattern of co-evolved residues preceding the CII domain (Figure 2B), consistent with a second

188 domain with a similar structure, henceforth referred to as the CI domain. When the structure of

189 the CI domain segment was predicted ${ }^{33,34}$ we found that it was also similar to the FlgT C-domain

190 (Figure 2A). This is distinct from previous predictions that identified only a single C-domain in

$191 \mathrm{TsaP}^{19}$.

192 The $\beta$-roll structure and extruding finger subdomain in the CI and CII models can be

193 clearly seen in the spoke density (Figure 2C). There is also density consistent with linker

194 residues preceding the CI domain and between the CI and CII domains. In contrast, Phyre ${ }^{2}$

195 predicted structures of PrpL and PA5122 do not fit the size or shape of the spokes. The C-

196 terminal 224 residues of TsaP encompassing the CI and CII domains were therefore modeled and

197 refined into the map. This model reveals that TsaP forms a heptameric ring with $\mathrm{C}_{7}$ symmetry

198 around $\mathrm{PilQ}^{\mathrm{Pa}}$ at the midpoint of the barrel where the internal gate is positioned (Figure 2D).

199 Each finger subdomain contacts the adjacent $\mathrm{C}$ domain to mediate intramolecular $\mathrm{CI}^{\mathrm{n}}-\mathrm{CII}^{\mathrm{n}}$

200 interactions and intermolecular $\mathrm{CII}^{\mathrm{n}}-\mathrm{CI}^{\mathrm{n}+1}$ interactions. These finger subdomain interactions

201 correlate with co-evolved residue pairs in TsaP (Figure 2B), further validating the model of TsaP

202 built into this map.

203 Each C domain contacts a separate PilQ protomer. The buried surface area of the TsaP-

204 PilQ interaction is $2924 \AA^{2}$ per TsaP protomer, or $20468 \AA^{2}$ for the entire 7:14 TsaP-PilQ

205 interaction. The buried surface area of the TsaP-TsaP oligomeric interface is $913 \AA^{2}$. The larger 
206 buried surface area of the TsaP-PilQ interface compared with the TsaP-TsaP oligomeric interface

207 hints that the PilQ interaction may help facilitate TsaP oligomerization.

\section{Overexpression of TsaP triggers cyclic di-GMP signalling}

211 trans by leaky expression from tsaP under an arabinose-inducible promotor without the addition

212 of arabinose (Figure 3A). We previously reported finding no obvious defect in twitching motility

213 with the $t s a P$ mutant ${ }^{18}$; this was likely the result of a limited number of replicates combined with

214 the modest phenotype. Compared to the vector control, a $66 \%$ defect in twitching motility is

215 observed in wild-type $P$. aeruginosa or the $t s a P$ mutant complemented with $t s a P$ when induced

216 with $0.1 \%(\mathrm{w} / \mathrm{v})$ arabinose - henceforth referred to as over-expressed TsaP (Figure 3B) -

217 consistent with both deletion and over-expression of TsaP impairing twitching motility.

218 We also assessed the propensity of $t s a P$ mutants and TsaP-overexpressing $P$. aeruginosa

219 to form biofilms, using a Congo red dye uptake assay. Congo red binds to the Pel polysaccharide

220 in P. aeruginosa biofilms, leading to red colonies and a small clear halo around the colonies

221 where the dye has been taken up ${ }^{35,36}$. We found that the $t s a P$ deletion mutant is indistinguishable

222 from wild-type $P$. aeruginosa (Figure 3B). However, TsaP overexpression in wild type or the

223 tsaP mutant leads to red colonies surrounded by pronounced clearing (Figure 3B), consistent

224 with the over-expression of TsaP leading to increased biofilm formation.

225 To look at other surface-associated phenotypes, we assessed the propensity of TsaP

226 overexpressing $P$. aeruginosa to secrete proteases using a casein proteolysis assay. Hydrolysis of

227 casein by extracelluar proteases leads to clear halos around protease- secreting colonies on solid

228 skim milk media ${ }^{37}$. Clear halos form around wild-type $P$. aeruginosa and the $t$ saP deletion

229 mutant; however, no halos form around TsaP over-expressing colonies (Figure 3B), consistent

230 with its over-expression decreasing extracellular protease secretion.

231 Reduced extracellular protease secretion, decreased twitching motility zones, and

232 increased biofilm formation are all associated with cyclic di-GMP induction ${ }^{4,38-40}$. Measurement

233 of luciferase expression from a cyclic di-GMP sensitive promotor verified that TsaP

234 overexpression increases cellular cyclic di-GMP concentrations (Figure 3C). Growth curves

235 confirmed that increased luciferase expression is not a result of increased cell growth (Figure 
236 3C). These results are consistent with TsaP over-expression up-regulating the production of 237 cyclic di-GMP.

\section{TsaP signalling occurs upstream of PilY1}

We probed the dependency of cyclic di-GMP signal upregulation caused by TsaP-

241 overexpression on other T4aP proteins. Surprisingly, deletion of PilQ or the PilQ pilotin, PilF,

242 did not prevent TsaP over-expression from increasing Congo-red dye uptake (Figure 3D). This

243 indicates that PilQ is not downstream of TsaP in this signal cascade. Like TsaP, PilY1 over-

244 expression in trans can trigger increased cyclic di-GMP production ${ }^{12}$. Deleting the pilMNOP

245 genes blocks cyclic di-GMP induction upon PilY1 over-expression ${ }^{12}$. When TsaP was over-

246 expressed in a pilY1 deletion mutant, there was decreased Congo-red dye uptake, consistent with

247 TsaP over-expression inducing a signal cascade that proceeds through PilY1 (Figure 3D).

248 Likewise, TsaP over-expression in pilN, pilO, or pilP mutants resulted in less Congo red dye 249 uptake (Figure 3D). These results are consistent with TsaP over-expression triggering a T4aP-

250 related cyclic di-GMP signal transduction pathway.

\section{DISCUSSION}

We determined the structure of PilQ alone and bound to TsaP, and found that TsaP over-

254 expression leads to increased expression of cyclic di-GMP, upstream of PilY1. Our results

255 resolve the identity of the spokes identified in Proteobacterial PilQ cryoEM densities and may

256 reveal a new component of the surface-sensing signal cascade.

257 Where most secretins are bound by their cognate pilotin around the periphery of the 258 secretin barrel adjacent to the secretin gate, we found $\mathrm{PilQ} \mathrm{Pa}^{\mathrm{Pa}}$ is bound by TsaP. Unlike pilotins, 259 TsaP is not essential for PilQ assembly or twitching motility ${ }^{16,18,19}$. Where and how the pilotin 260 PilF interacts with PilQ remains an open question, although one possibility is that TsaP replaces 261 PilF after insertion of PilQ into the outer membrane. We identified co-evolved residues that are 262 consistent with the TsaP-binding region of PilQ interacting with the hydrophobic membrane263 interacting interface. This hints at a model of PilQ membrane insertion in which secretin 264 monomers initially fold onto themselves, with the TsaP-binding site occluding the hydrophobic 265 membrane insertion site. Binding by pilotins to the TsaP-binding site might then promote the 266 insertion of PilQ into the membrane, rationalizing the necessity of pilotins for secretin assembly 
267 and localization. If this were the case, after outer-membrane insertion of PilQ, PilF could be 268 replaced by TsaP.

269 It seems likely that TsaP and PilQ are tightly associated, as the buried surface area of the

270 TsaP-PilQ interaction is enormous $\left(20468 \AA^{2}\right)$ and the complex is stable in detergent. It is

271 nonetheless possible that some of the TsaP rings were removed during the purification, as only

272 about half of the purified PilQ particles have TsaP associated with them. In this case, PilQ

273 partially bound by TsaP might be expected, but only PilQ entirely bound by TsaP or without

274 TsaP were identified in cryoEM 3D classes. Alternatively, there may be a population of free PilQ

275 oligomers representing an intermediate state between PilQ-PilF and PilQ-TsaP complexes.

276 The structure of PilQ was determined in two distinct stoichiometries. Interestingly, only

277 the tetradecameric form is bound by TsaP and had the unidentified secretin gate plug. The 2:1

278 PilQ:TsaP configuration necessitates binding of only even-numbered PilQ stoichiometries. It may

279 be that TsaP recognizes or selects for specific secretin stoichiometries. Selecting particular

280 secretin stoichiometries may improve the efficiency of T4aP function, consistent with the

281 moderate twitching motility defect of the TsaP-deletion mutant.

282 In addition to influencing the quaternary structure of PilQ, TsaP binding may alter its

283 tertiary structure. Comparing the different PilQ structures revealed a pivot point for secretin gate

284 invagination adjacent to the spokes, consistent with TsaP binding influencing this process. Thus,

285 a plausible role for TsaP may be in modifying secretin-gate invagination. The consequences of

286 this are not clear, but the gate could then interact with the pilus initiation complex of minor pilins

287 and PilY1 and/or the unidentified secretin gate plug.

288 TsaP could also be involved with larger-scale secretin movements. For example, PilQ

289 adopts distinct open and closed states depending on whether the pilus fibre occupies the central

290 channel the $\mathrm{T}_{4} \mathrm{aP}^{19,20}$. Compared to $\mathrm{T} 4 \mathrm{aP}$ maps obtained by electron cryotomography in which

291 the pilus fibre does not occupy the T4aP, the OM in the piliated state has moved $20 \AA$ away from

292 PilP and the peptidoglycan ${ }^{19}$. Although the secretin domain is embedded in the OM, the AMI_N

293 and N0 domains of PilQ bind peptidoglycan and PilP, respectively ${ }^{41}$. This suggests that PilQ

294 must extend and contract $20 \AA$ between the piliated and non-piliated states. As TsaP binds to both

295 the peptidoglycan and secretin domain of PilQ, TsaP binding could modulate secretin opening.

296 For example, TsaP could act as an elastic band between the peptidoglycan and outer-membrane

297 components of PilQ, keeping PilQ contracted and the T4aP closed in the absence of an assembled 
pilus. The predicted disordered residues between the LysM and CI/CII domains could afford TsaP the elasticity required for this action. and relay this information as a part of a signal cascade. However, while TsaP over-expression triggers the cyclic di-GMP signal cascade, it does so independently of PilQ. This is curious as PilQ provides the only known physical contact between TsaP and PilN, PilO, and PilP - which are important for inducing cyclic di-GMP production. It may be that there is a PilQ-independent signal transduction pathway from TsaP to PilY1 and PilNOP. Alternatively, over-expressing TsaP may force a signalling event that is typically dependent on PilQ. As TsaP has a peptidoglycan-binding LysM domain, overexpression might place strain on the peptidoglycan that resembles what happens during surface interactions, leading to cyclic di-GMP induction. In the absence of a known trigger for the signalling cascade upstream of TsaP, it is not clear what role TsaP plays in the surface-sensing signal cascade under native expression conditions.

311 Nonetheless, knowing that TsaP over-expression can trigger the cyclic di-GMP signal cascade 312 upstream of PilY1 will be useful in identifying other key players in the surface sensing signal 313 cascade.

\section{METHODS}

\section{Cloning and mutagenesis}

317 TsaP from $P$. aeruginosa PAO1 was PCR amplified from genomic DNA using primers 318 TsaP_fwd and TsaP_rev (Supplementary Table 3), digested with KpnI and XbaI, and cloned into 319 pBADGr to create pBADGr:tsaP for arabinose inducible expression of TsaP. Plasmid sequences 320 were verified by TCAG sequencing facilities (The Hospital for Sick Children, Canada).

\section{TsaP Deletion}

A gene deletion allele was ordered from BioBasic and subcloned into pEX18Gm at the 324 BamHI site producing pEX18Gm_tsaP. Clones were confirmed by digest and DNA sequencing 325 (TCAG, The Hospital for Sick Children). The pEX18Gm_tsaP construct was transformed into 326 chemically competent SM10 E. coli cells, colonies containing pEX18Gm_tsaP were mated with 327 mPAO1 using a method described elsewhere ${ }^{42}$. Briefly, after mating $P$. aeruginosa was selected 328 for by growth on LB agar plates containing $25 \mu \mathrm{g} / \mathrm{ml}$ triclosan (Sigma) followed by selection of 
329 the merodiploid strains on VBMM agar containing $30 \mu \mathrm{g} / \mathrm{ml}$ gentamicin. Counter selection to 330 resolve the merodiploids was performed on $1.5 \%$ agar plates with $10 \mathrm{~g} / \mathrm{L}$ tryptone, $5 \mathrm{~g} / \mathrm{L}$ yeast 331 extract, and 15\% (wt/vol) sucrose. The deletion was verified by the absence of a PCR product using 332 primers TsaP_fwd and TsaP_rev.

\section{Phenotypic characterizations}

335 P. aeruginosa PAO1 with and without deletion mutations of $t s a P$, pilN, pilO, pilP, pilQ, 336 pilY1 were electroporated with pBADGr or pBADGr:tsaP. P. aeruginosa PAO1 with and without 337 deletion mutations of $t s a P$ were also electroporated with pBADGr:tsaP.

338 Twitching assays were performed as described previously ${ }^{43,44}$ except that assays were 339 performed in $150 \mathrm{~mm}$ by $15 \mathrm{~mm}$ petri polystyrene dishes (Fisher Scientific) with $30 \mu \mathrm{g} / \mathrm{ml}$ 340 gentamicin for $18 \mathrm{~h}$ at $37^{\circ} \mathrm{C}$. After this incubation, the agar was then carefully discarded and the 341 adherent bacteria were stained with $1 \%(\mathrm{w} / \mathrm{v})$ crystal violet dye, followed by washing with 342 deionized water to remove unbound dye. Twitching zone areas were measured using ImageJ 343 software ${ }^{45}$. Twitching motility assays were performed in six replicates.

$344 \quad$ For Congo-red dye binding assays, cells were grown in $0.5 \mathrm{~mL}$ of lysogeny broth (LB) with $34530 \mu \mathrm{g} / \mathrm{ml}$ gentamicin for $16 \mathrm{~h}$ at $37^{\circ} \mathrm{C}$ while shaking. Cultures were normalized to an OD at 600 $346 \mathrm{~nm}$ of 0.1 and then $3 \mu \mathrm{l}$ of culture was spotted onto Congo red plates ( $1 \%(\mathrm{wt} / \mathrm{vol})$ Tryptone, $1 \%$ 347 (wt/vol) agar, 0.004\% (wt/vol) congo red, and 0.0015\% (wt/vol) Coomassie Brilliant Blue R250, 348 and either $0,0.01$, or $0.1 \%$ L-arabinose) with the appropriate antibiotic, and grown for $24-36 \mathrm{~h}$ at $34937^{\circ} \mathrm{C}$.

350 Extracellular proteases were assayed using skim milk plates (1\% wt/vol agar, $1 \% \mathrm{wt} / \mathrm{vol}$ 351 skim milk powder, and either $0,0.01$, or $0.1 \%$ L-arabinose) with the appropriate antibiotic, using 352 the same normalized cultures used for the Congo red binding assays. $3 \mu \mathrm{l}$ of culture was spotted 353 on a plate and incubated for $16 \mathrm{hrs}$ at $37^{\circ} \mathrm{C}$. All plates were scanned using an Epson Perfection 3544990 scanner and Epson Scan software.

355 For luciferase expression, cells previously electroporated with pBADGr, pBADGr::tsaP 356 and pBADGr::tsaP mutant constructs were electroporated with pMS402:PcdrA. Cells were grown 357 in $0.5 \mathrm{~mL}$ of lysogeny broth (LB) with $30 \mu \mathrm{g} / \mathrm{ml}$ gentamicin and $150 \mu \mathrm{g} / \mathrm{ml}$ kanamycin for $16 \mathrm{~h}$ at $35837^{\circ} \mathrm{C}$ while shaking. Cultures were normalized to OD600 of 0.1 and diluted 10x into fresh LB 359 broth containing $30 \mu \mathrm{g} / \mathrm{ml}$ gentamicin, $105 \mu \mathrm{g} / \mathrm{ml}$ kanamycin and either $0,0.01 \%$ or $0.1 \% \mathrm{~L}$ - 
360 arabinose in a 384 well plate (Corning, 3821). All samples were done in triplicate. Growth and

361 luciferase activity were monitored over a 16-hour period in a Synergy Neo2 plate reader (BioTek

362 Instruments). Data was analyzed and plotted using Prism 8 (Graph Pad).

Plotting the residues at the packing unit interface

$\mathrm{CMview}^{46}$ was used to identify residues that are near one another. The contact type was set to 'ALL' (i.e. every atom available in the structure) and the distance cut-off was set to $8 \AA$. To identify residues that are near one another in PilQ monomers, the CI domain of TsaP (residues 126211), or the CII domain of TsaP (residues 235-324), these structure segments were loaded

369 separately. To identify intra-molecular CI to CII residues that are near each other in TsaP, full-

370 length TsaP was loaded. To identify inter-molecular residues that are near one another, two

371 adjacent PilQ protomers were loaded together, or the CI domain and CII domains from two adjacent

372 protomers were loaded together.

373

\section{Identifying evolutionarily-coupled residues}

375 The $\mathrm{TsaP}$ and $\mathrm{PilQ}^{\mathrm{Pa}}$ amino acid sequences were analyzed using the EVcouplings 376 webserver ${ }^{26}$ with default parameters. Homologs were identified, aligned, and analyzed - in total, 377 2642 and 11302 for TsaP and PilQ, respectively. There was poor alignment quality in the lip 378 subdomain of PilQ (residues 481-531) so few evolutionarily-coupled residue pairs were discovered

379 in the lip subdomain. Only residue pairs with a probability score greater than 0.845 or 0.985 were included in subsequent analysis, for TsaP and PilQ, respectively. To better understand the

381 significance of these evolutionarily coupled residues, they were compared to the residues that are 382 near one another identified in CMview.

\section{CryoEM analysis}

As previously described for this dataset ${ }^{18}$, cryoEM data was collected as movies ( 30 frames over $15 \mathrm{sec}$ ) with a FEI Tecnai F20 electron microscope operating at $200 \mathrm{kV}$ and equipped with a

387 Gatan K2 Summit direct detector camera. The exposure rate was 5 electrons/pixel/s with a 388 calibrated pixel size of $1.45 \AA$ /pixel. Previously, movie frames had been aligned with 389 alignframes_lmbfgs ${ }^{47}$ and then individual movie frames were discarded for data storage purposes; 390 this prevented exposure weighting and individual particle motion correction herein. Further image 
391 processing here was performed in cryoSPARC v2.8.0 24 (Supplementary Table 1). CTF

392 parameters were estimated from the average of aligned frames with CTFFIND4 ${ }^{48}$ within 393 cryoSPARC and individual particle motion correction and exposure weighting was done with an

394 implementation of alignparts_lmbfgs ${ }^{47}$ within cryoSPARC. Initial 2D class averages were 395 generated with manually selected particle images; these class averages were then used to select 396 more particle images. Particle images were extracted in $256 \times 256$ pixel boxes then subjected to 2D 397 classification. For the purposes of 3D classification, particle images contributing to 2D classes 398 without high-resolution features were removed. Ab initio reconstruction was performed using two 399 classes, revealing barrels and spoked-barrels. The particles contributing to the barrels without 400 spokes were used for an additional round of ab initio reconstruction with two classes, revealing an 401 additional class with wider barrels. The spoked barrels, the barrels without spokes, and the wider 402 barrels without spokes were then used as initial models for heterogeneous refinement; particles 403 from 3D classes that did not converge at this stage were removed. Particles from distinct 3D classes 404 were then subjected to non-uniform refinement ${ }^{49}$. The resulting fourier shell correlations for the 4054.3 and $4.4 \AA$ maps are unusual as there is a flat region between $\sim 7 \AA$ and $\sim 5 \AA$. This likely arises 406 from the diffuse density from the detergent layer and the N-terminal domains disordered relative 407 to the secretin of PilQ. However, the clear features from the beta-barrels support the resolution 408 estimates for the maps

A molecular model could be built using $\operatorname{Coot}^{50}$ into the spoked barrel map using the 411 structures of FlgT and GspD 25,32 as guides for TsaP and PilQ, respectively. Molecular models for 412 the maps of barrels without spokes were built by fitting the PilQ protomers built into the spoked 413 barrel map in $\operatorname{Coot}^{50}$. These models were refined against the maps in Phenix-Refine ${ }^{51}$ with 414 reference model restraints to the spoked barrel PilQ structures. While side-chains were used to 415 ensure the models made biological sense during model building, they were ultimately removed as 416 the map quality did not justify them being modeled.

\section{Acknowledgements}

420 This work was supported by a grant from the Canadian Institutes for Health Research (CIHR) MOP 42193585 to L.L.B. and P.L.H. M.M. was supported by a CIHR Doctoral Studentship and Ontario 
422 Graduate Scholarship during these studies. P.L.H. and J.L.R. are recipients of Canada Research

423 Chairs. The graphics programs used in this study were accessed using SBGrid.

424

425

426

\section{REFERENCES}

427 1. McCallum, M., Burrows, L.L. \& Howell, P.L. The Dynamic Structures of the Type IV

428 Pilus. Microbiol Spectrum 7(2019).

429 2. Craig, L., Forest, K.T. \& Maier, B. Type IV pili: dynamics, biophysics and functional

$430 \quad$ consequences. Nat Rev Microbiol 17, 429-440 (2019).

431 3. Chang, C.Y. Surface Sensing for Biofilm Formation in Pseudomonas aeruginosa. Front

$432 \quad$ Microbiol 8, 2671 (2017).

433 4. Valentini, M. \& Filloux, A. Biofilms and Cyclic di-GMP (c-di-GMP) Signaling: Lessons

434 from Pseudomonas aeruginosa and Other Bacteria. J Biol Chem 291, 12547-55 (2016).

435 5. Mah, T.F. \& O'Toole, G.A. Mechanisms of biofilm resistance to antimicrobial agents.

436 Trends Microbiol 9, 34-9 (2001).

437 6. McCallum, M., Tammam, S., Khan, A., Burrows, L.L. \& Howell, P.L. The molecular

438

439

440

441

442

443

444

445

446

447

448

449

450

451

452

453 mechanism of the type IVa pilus motors. Nat Commun 8, 15091 (2017).

7. McCallum, M. et al. Multiple conformations facilitate PilT function in the type IV pilus. Nat Commun 10, 5198 (2019).

8. Marko, V.A., Kilmury, S.L.N., MacNeil, L.T. \& Burrows, L.L. Pseudomonas aeruginosa type IV minor pilins and PilY1 regulate virulence by modulating FimS-AlgR activity. PLoS Pathog 14, e1007074 (2018).

9. Nguyen, Y. et al. Pseudomonas aeruginosa minor pilins prime type IVa pilus assembly and promote surface display of the PilY1 adhesin. J Biol Chem 290, 601-11 (2015).

10. McCallum, M. et al. PilN Binding Modulates the Structure and Binding Partners of the Pseudomonas aeruginosa Type IVa Pilus Protein PilM. J Biol Chem 291, 11003-15 (2016).

11. Tammam, S. et al. PilMNOPQ from the Pseudomonas aeruginosa type IV pilus system form a transenvelope protein interaction network that interacts with PilA. J Bacteriol 195, 2126-35 (2013).

12. Luo, Y. et al. A hierarchical cascade of second messengers regulates Pseudomonas aeruginosa surface behaviors. mBio 6(2015).

454

455

456

457

458

459

460

461

462

463

464

13. Persat, A., Inclan, Y.F., Engel, J.N., Stone, H.A. \& Gitai, Z. Type IV pili mechanochemically regulate virulence factors in Pseudomonas aeruginosa. Proc Natl Acad Sci U S A 112, 7563-8 (2015).

14. Filloux, A. \& Voulhoux, R. Multiple Structures Disclose the Secretins' Secrets. $J$ Bacteriol 200(2018).

15. Worrall, L.J. et al. Near-atomic-resolution cryo-EM analysis of the Salmonella T3S injectisome basal body. Nature 540, 597-601 (2016).

16. Siewering, K. et al. Peptidoglycan-binding protein TsaP functions in surface assembly of type IV pili. Proc Natl Acad Sci U S A 111, E953-61 (2014).

17. Collins, R.F. et al. Structure of the Neisseria meningitidis outer membrane PilQ secretin complex at 12 A resolution. J Biol Chem 279, 39750-6 (2004). 
18. Koo, J., Lamers, R.P., Rubinstein, J.L., Burrows, L.L. \& Howell, P.L. Structure of the Pseudomonas aeruginosa Type IVa Pilus Secretin at 7.4 A. Structure 24, 1778-1787 (2016).

19. Chang, Y.W. et al. Architecture of the type IVa pilus machine. Science 351, aad2001 (2016).

20. Weaver, S.J., Sazinsky, M.H., Dalia, T.N., Dalia, A.B. \& Jensen, G.J. CryoEM structure

471

472

473 of the Vibrio cholerae Type IV competence pilus secretin PilQ. bioRxiv (2020).

21. D'Imprima, E. et al. Cryo-EM structure of the bifunctional secretin complex of Thermus thermophilus. Elife 6, e30483 (2017).

22. Yin, M., Yan, Z. \& Li, X. Structural insight into the assembly of the type II secretion system pilotin-secretin complex from enterotoxigenic Escherichia coli. Nat Microbiol 3, 581-587 (2018).

23. Chernyatina, A.A. \& Low, H.H. Core architecture of a bacterial type II secretion system. Nat Commun 10, 5437 (2019).

24. Punjani, A., Rubinstein, J.L., Fleet, D.J. \& Brubaker, M.A. cryoSPARC: algorithms for rapid unsupervised cryo-EM structure determination. Nat Methods 14, 290-296 (2017).

25. Yan, Z., Yin, M., Xu, D., Zhu, Y. \& Li, X. Structural insights into the secretin translocation channel in the type II secretion system. Nat Struct Mol Biol 24, 177-183 (2017).

26. Marks, D.S. et al. Protein 3D structure computed from evolutionary sequence variation. PLoS One 6, e28766 (2011).

27. de Juan, D., Pazos, F. \& Valencia, A. Emerging methods in protein co-evolution. Nat Rev Genet 14, 249-61 (2013).

28. $\mathrm{Hu}$, J. et al. Cryo-EM analysis of the T3S injectisome reveals the structure of the needle and open secretin. Nat Commun 9, 3840 (2018).

29. Nudleman, E., Wall, D. \& Kaiser, D. Polar assembly of the type IV pilus secretin in Myxococcus xanthus. Mol Microbiol 60, 16-29 (2006).

30. Wilderman, P.J. et al. Characterization of an endoprotease (PrpL) encoded by a PvdSregulated gene in Pseudomonas aeruginosa. Infect Immun 69, 5385-94 (2001).

31. Michel, G.P., Durand, E. \& Filloux, A. XphA/XqhA, a novel GspCD subunit for type II secretion in Pseudomonas aeruginosa. J Bacteriol 189, 3776-83 (2007).

32. Terashima, H. et al. Insight into the assembly mechanism in the supramolecular rings of the sodium-driven Vibrio flagellar motor from the structure of FlgT. Proc Natl Acad Sci U S A 110, 6133-8 (2013).

33. Kelley, L.A., Mezulis, S., Yates, C.M., Wass, M.N. \& Sternberg, M.J. The Phyre2 web portal for protein modeling, prediction and analysis. Nat Protoc 10, 845-58 (2015).

34. Yang, J. et al. The I-TASSER Suite: protein structure and function prediction. Nat Methods 12, 7-8 (2015).

35. Jones, C.J. \& Wozniak, D.J. Congo Red Stain Identifies Matrix Overproduction and Is an Indirect Measurement for c-di-GMP in Many Species of Bacteria. Methods Mol Biol 1657, 147-156 (2017).

36. Vasseur, P., Vallet-Gely, I., Soscia, C., Genin, S. \& Filloux, A. The pel genes of the Pseudomonas aeruginosa PAK strain are involved at early and late stages of biofilm formation. Microbiology 151, 985-997 (2005).

37. Sokol, P.A., Ohman, D.E. \& Iglewski, B.H. A more sensitive plate assay for detection of protease production by Pseudomanas aeruginosa. J Clin Microbiol 9, 538-40 (1979). 
511 38. Ravichandran, A., Ramachandran, M., Suriyanarayanan, T., Wong, C.C. \& Swarup, S.

512

513

514

515

516

517

518

519

520

521

522

523

524

525

526

527

528

529

530

531

532

533

534

535

536

537

538

539

540

541

542

543

544

545

546

547

548

549

550

551

552

Global Regulator MorA Affects Virulence-Associated Protease Secretion in Pseudomonas aeruginosa PAO1. PLoS One 10, e0123805 (2015).

39. Lopez-Baena, F.J., Vinardell, J.M. \& Medina, C. Regulation of Protein Secretion Systems Mediated by Cyclic Diguanylate in Plant-Interacting Bacteria. Front Microbiol 10, 1289 (2019).

40. Jain, R., Behrens, A.J., Kaever, V. \& Kazmierczak, B.I. Type IV pilus assembly in Pseudomonas aeruginosa over a broad range of cyclic di-GMP concentrations. J Bacteriol 194, 4285-94 (2012).

41. Berry, J.L. et al. Structure and assembly of a trans-periplasmic channel for type IV pili in Neisseria meningitidis. PLoS Pathog 8, e1002923 (2012).

42. de Lorenzo, V. \& Timmis, K.N. Analysis and construction of stable phenotypes in gramnegative bacteria with Tn5- and Tn10-derived minitransposons. Methods Enzymol 235, 386-405 (1994).

43. Ha, D.G., Kuchma, S.L. \& O'Toole, G.A. Plate-based assay for swarming motility in Pseudomonas aeruginosa. Methods Mol Biol 1149, 67-72 (2014).

44. Turnbull, L. \& Whitchurch, C.B. Motility assay: twitching motility. Methods Mol Biol 1149, 73-86 (2014).

45. Schneider, C.A., Rasband, W.S. \& Eliceiri, K.W. NIH Image to ImageJ: 25 years of image analysis. Nat Methods 9, 671-5 (2012).

46. Vehlow, C. et al. CMView: interactive contact map visualization and analysis. Bioinformatics 27, 1573-4 (2011).

47. Rubinstein, J.L. \& Brubaker, M.A. Alignment of cryo-EM movies of individual particles by optimization of image translations. J Struct Biol 192, 188-95 (2015).

48. Rohou, A. \& Grigorieff, N. CTFFIND4: Fast and accurate defocus estimation from electron micrographs. J Struct Biol 192, 216-21 (2015).

49. Punjani, A., Zhang, H., Fleet D. J. Non-uniform refinement: Adaptive regularization improves single particle cryo-EM reconstruction. bioRxiv (2019).

50. Casanal, A., Lohkamp, B. \& Emsley, P. Current Developments in Coot for Macromolecular Model Building of Electron Cryo-microscopy and Crystallographic Data. Protein Sci (2019).

51. Afonine, P.V. et al. Real-space refinement in PHENIX for cryo-EM and crystallography. Acta Crystallogr D Struct Biol 74, 531-544 (2018).

\section{Figure Legends}

Figure 1. Electron cryomicroscopy of PilQ from $P$. aeruginosa reveals multiple conformers. A-C, Map (upper left) and model (upper right) of $\mathrm{PilQ}^{\mathrm{Pa}}$ (coloured) with additional spoke and plug density (black). Top (top), side (middle), and cross section (bottom) views are shown. A, Map and model of spoked tetradecameric PilQ ${ }^{\mathrm{Pa}}$ (purple). B, Map and model of tetradecameric PilQ ${ }^{\mathrm{Pa}}$ without spokes (teal). C, Map and model of pentadecameric PilQ ${ }^{\mathrm{Pa}}$ (orange). D,

Evolutionarily coupled residues in PilQ (gray) are compared with the structure of PilQ ${ }^{\mathrm{Pa}}$. Residue 
553 pairs that are within $8 \AA$ of each other in the tertiary structure of $\mathrm{PilQ}^{\mathrm{Pa}}$ are shown (black).

554 Residue pairs that are within $8 \AA$ of each other between PilQ ${ }^{\mathrm{Pa}}$ protomers are also shown (gold).

555 Specific evolutionarily coupled residues that are not supported by the PilQ ${ }^{\mathrm{Pa}}$ structure are noted

556 with coloured arrows: G554-P635 (yellow), E663-A624 (red), T538-E652 (green), L545-T653

557 (magenta), and L546-V457 (blue). E, Location of the G554-P635 and E663-A624 residue pairs,

558 as well as hinge residue G577 (asterisk), on a cross section of PilQ ${ }^{\mathrm{Pa}}$ (left) and a model for

559 secretin gate opening consistent with these co-evolved residues. F, Location of the T538-E652,

560 L545-T653, and L546-V457 residue pairs on PilQ ${ }^{\mathrm{Pa}}$ (left) and a model for secretin barrel folding

561 consistent with these co-evolved residues. G, Surface representation of conserved residues (top)

562 and electrostatic potential (bottom) in $\mathrm{PilQ}^{\mathrm{Pa}}$. H, Structure alignment of monomers from the

563 spoked tetradecameric $\mathrm{PilQ}^{\mathrm{Pa}}$ (purple), tetradecameric $\mathrm{PilQ}^{\mathrm{Pa}}$ with no spokes (teal), and

564 pentadecameric PilQ ${ }^{\mathrm{Pa}}$ (orange) models. Grey square with black PilQ cartoon has a dotted red

565 box highlighting area of PilQ shown. Structural differences are highlighted (arrows) and the pivot

566 point for these differences is noted (black circle). I, Cartoon model summarizing the differences

567 between the three $\mathrm{PilQ}^{\mathrm{Pa}}$ structures determined. Pentadecameric PilQ ${ }^{\mathrm{Pa}}$ (orange) is wider and has

568 more horizontal secretin gates compared to the other structures. Tetradecameric PilQ ${ }^{\mathrm{Pa}}$ (teal) has

569 invaginated secretin gates and partial plug density (black triangle). Spoked tetradecameric PilQ ${ }^{\mathrm{Pa}}$

570 (purple) has further invaginated secretin gates, plug density (black triangle), and spokes (black

571 circles).

572

573 Figure 2. TsaP can be modeled in the PilQ spoke density as a heptameric ring. A, TsaP

574 domain model predictions: the I-Tasser prediction of the CI domain (residues 126-211) ${ }^{34}$ and the

575 Phyre $^{2}$ prediction of the CII domain (residues 235-324) ${ }^{33}$. B, Evolutionarily coupled residues in

576 TsaP (gray) are compared with the structure of the C-terminal 224 residues of TsaP. Residue

577 pairs that are within $8 \AA$ of each other are shown from the CI domain (yellow), the CII domain

578 (wheat-yellow), and the linkers preceding and following the CI domain (brown). Intramolecular

579 and intermolecular contacts in the TsaP structure between the CI and CII domains are also shown

580 (blue and red, respectively). C-D, Map (top), model (middle), and cartoon (bottom) summarizing

581 the structure of TsaP. Zoomed-in $(\mathbf{C})$ and zoomed-out (D) views are shown. TsaP is coloured as

582 in B; PilQ is shown in grey $(\mathbf{C})$ or purple (D).

583 
584 Figure 3. TsaP over-expression induces cyclic di-GMP up-regulation. Wild-type P. aeruginosa

585 (left), the tsaP mutant (center left), P. aeruginosa expressing TsaP (center right), and the tsaP

586 mutant expressing TsaP (right). A, Twitching motility was assayed by crystal violet staining and

587 the areas traversed by the bacteria were measured (violin plot with individual measurements shown

588 as empty circles; $\mathrm{n}=7$ for Wild-type with and without vector, $\mathrm{n}=6$ for the $t$ saP mutant with and

589 without vector; data are representative of three independent trials). Mean twitching areas were

590 compared to wild type plus the control vector at the corresponding arabinose concentration with

591 the two-way ANOVA test; p-values are reported. B, Representative crystal violet stained twitching

592 motility zones (top), Congo-red agar colonies (middle), and skim milk agar colonies (bottom) are

593 shown for $0 \%$ arabinose induction (-) or $0.1 \%$ arabinose induction $(++)$. $\mathbf{C}$, Luminescence over

594 time relative to uninduced control without arabinose (top), with luciferase expression under the

595 control of the cyclic di-GMP inducible $c d r A$ promotor. Mean luminescence ( $\mathrm{n}=3$, representative of

596 three independent trials) relative to a matched uninduced control is shown for $0.1 \%$ arabinose

597 induction (orange, ++ ) or $0.01 \%$ arabinose induction (beige, + ) as a filled in line with the standard

598 error of the mean (dotted lines). Growth measured by absorbance at $600 \mathrm{~nm}$ over time of liquid

599 cultures (bottom). Mean growth ( $n=3$, representative of three independent trials) is shown for $0 \%$

600 arabinose induction (gray, -), $0.01 \%$ arabinose induction (beige, + ), or $0.1 \%$ arabinose induction

601 (orange, ++ ) with the standard error of the mean shown (dotted lines). D, Congo red dye uptake

602 assay of PAO1 (wild-type), PAO1::pilN, PAO1::pilO, PAO1::pilP, PAO1::pilQ, PAO1::pilA,

603 PAO1::pilF and PAO1::pilY1 with (++) and without (-) TsaP overexpression.

604

605

606 
bioRxiv preprint doi: https://doi.org/10.1101/2020.05.29.123786; this version posted September 1, 2020. The copyright holder for this preprint (which was not certified by peer review) is the author/funder, who has granted bioRxiv a license to display the preprint in perpetuity. It is made available under aCC-BY-NC-ND 4.0 International license.

607 Figure 1

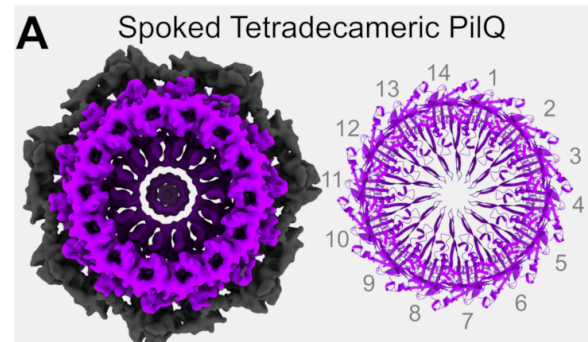

$\rightarrow 90^{\circ}$
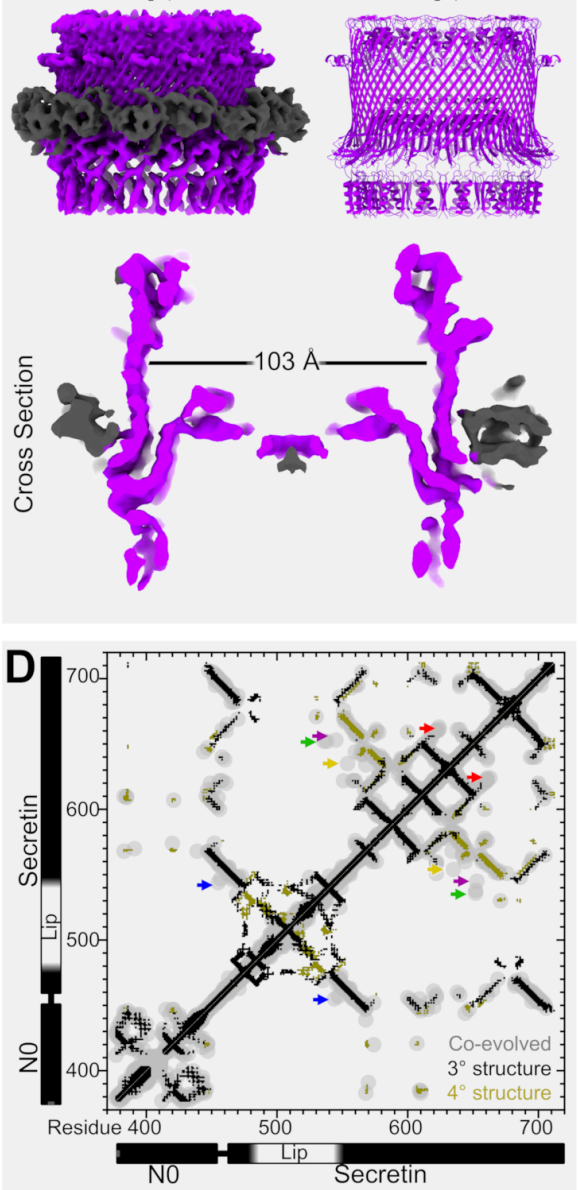

\section{G}

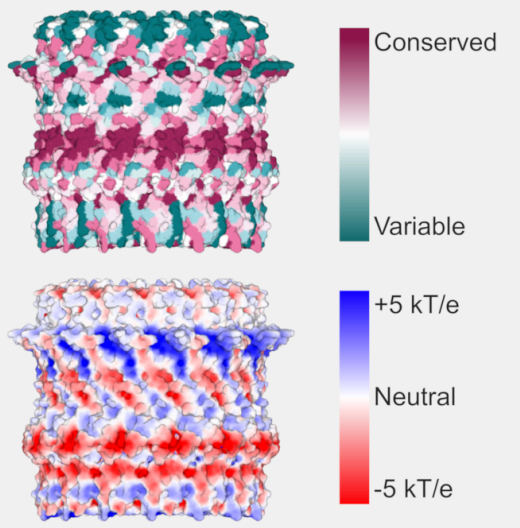

B Tetradecameric PilQ

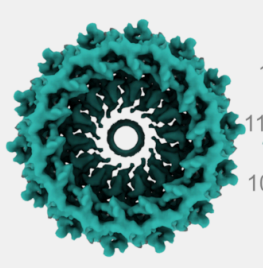

ㄱ. $90^{\circ}$
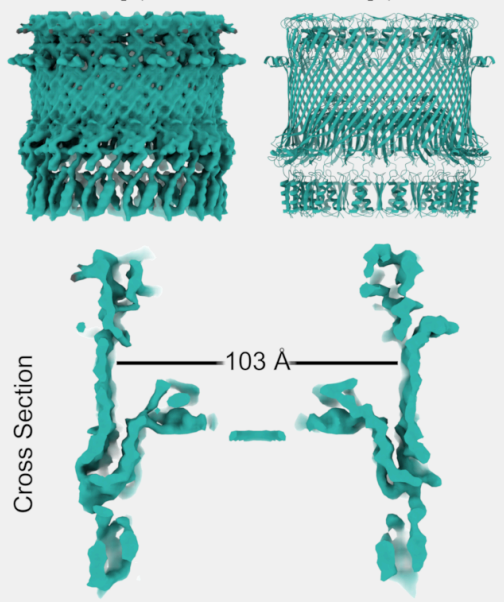

C Pentadecameric PilQ

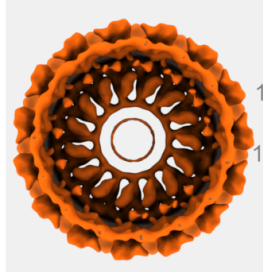

ก) $90^{\circ}$
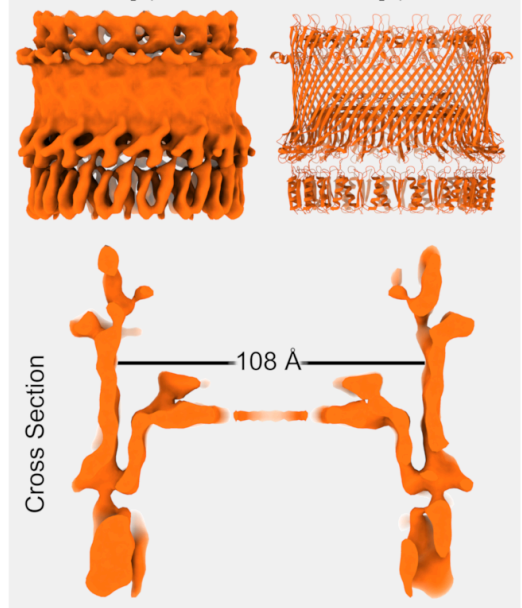
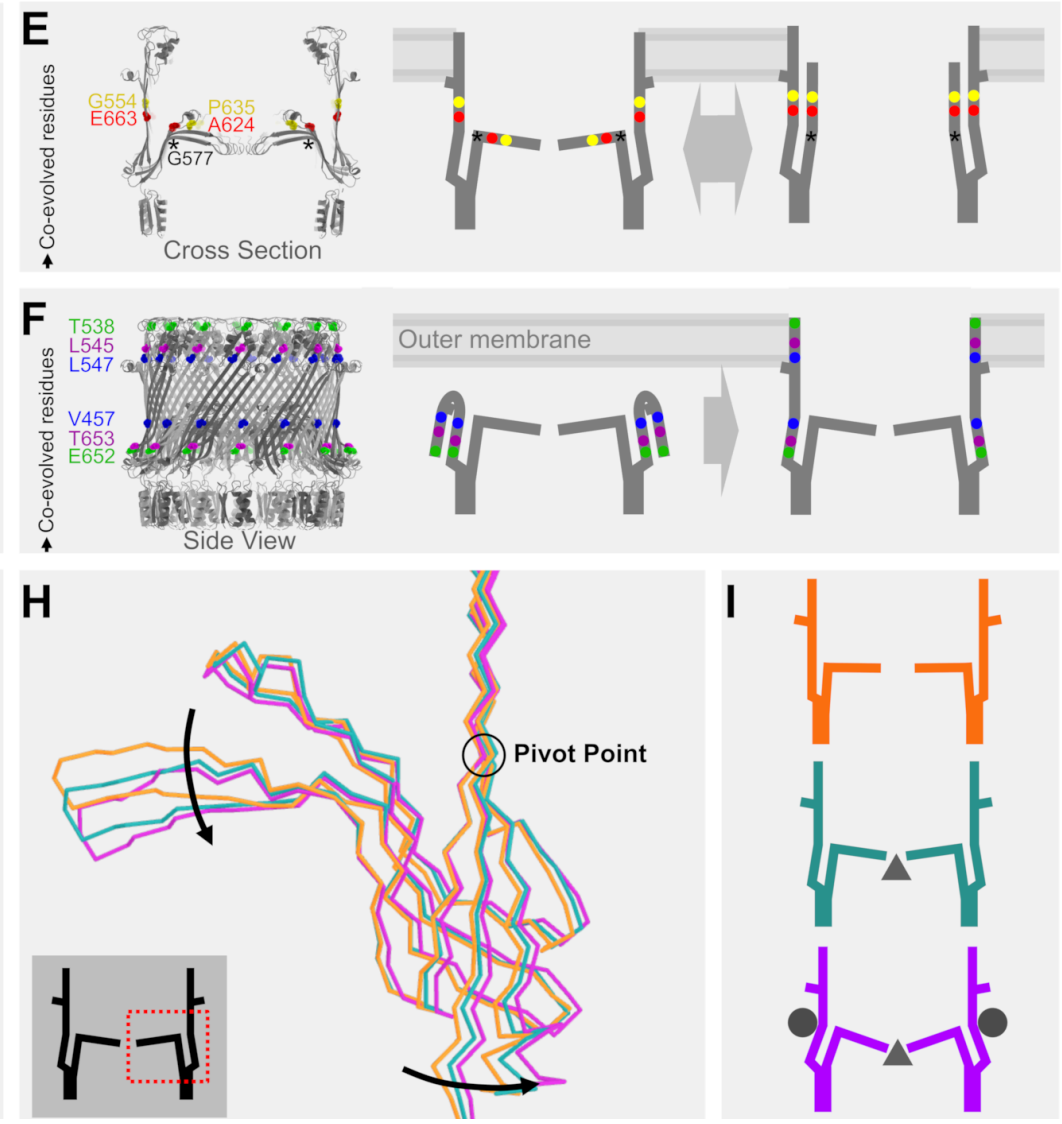
bioRxiv preprint doi: https://doi.org/10.1101/2020.05.29.123786; this version posted September 1, 2020. The copyright holder for this preprint (which was not certified by peer review) is the author/funder, who has granted bioRxiv a license to display the preprint in perpetuity. It is made available under aCC-BY-NC-ND 4.0 International license.

609 Figure 2

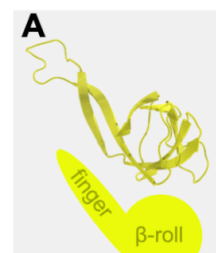

CI Prediction

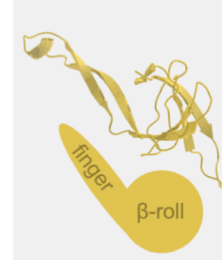

CII Prediction

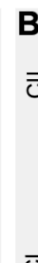

${ }^{3}{ }^{30}$

$\overline{0}$

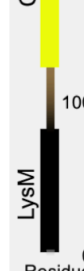

Residue

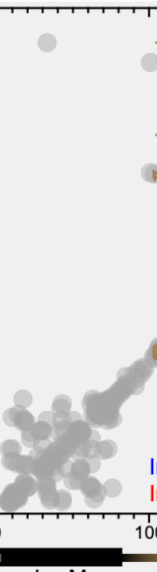

LysM

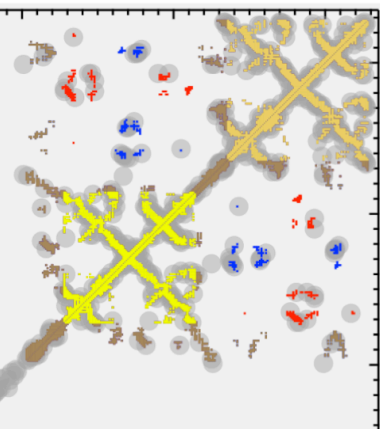

Co-evolved Residues ntramolecular $\mathrm{Cl}-\mathrm{Cll}$ Contacts Intermolecular CII-CI Contacts 100 200 Cl
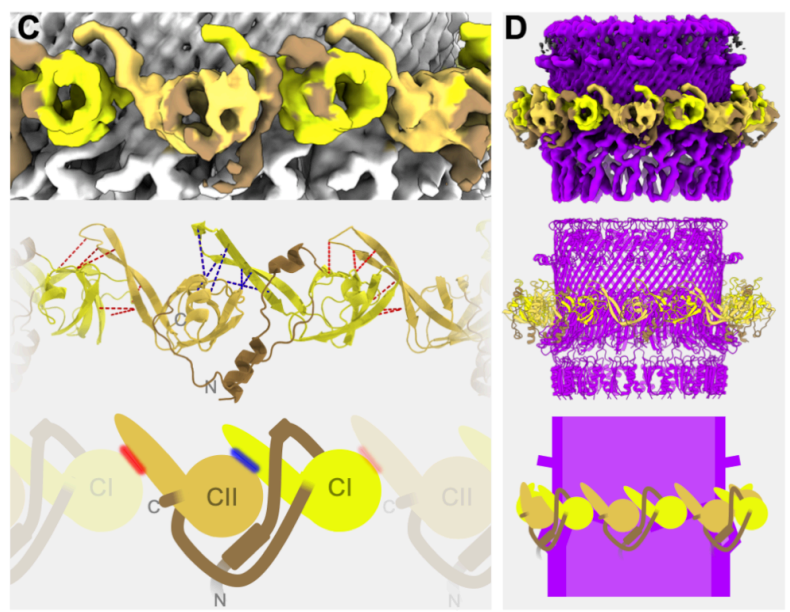
bioRxiv preprint doi: https://doi.org/10.1101/2020.05.29.123786; this version posted September 1, 2020. The copyright holder for this preprint (which was not certified by peer review) is the author/funder, who has granted bioRxiv a license to display the preprint in perpetuity. It is made available under aCC-BY-NC-ND 4.0 International license.

\section{Figure 3}

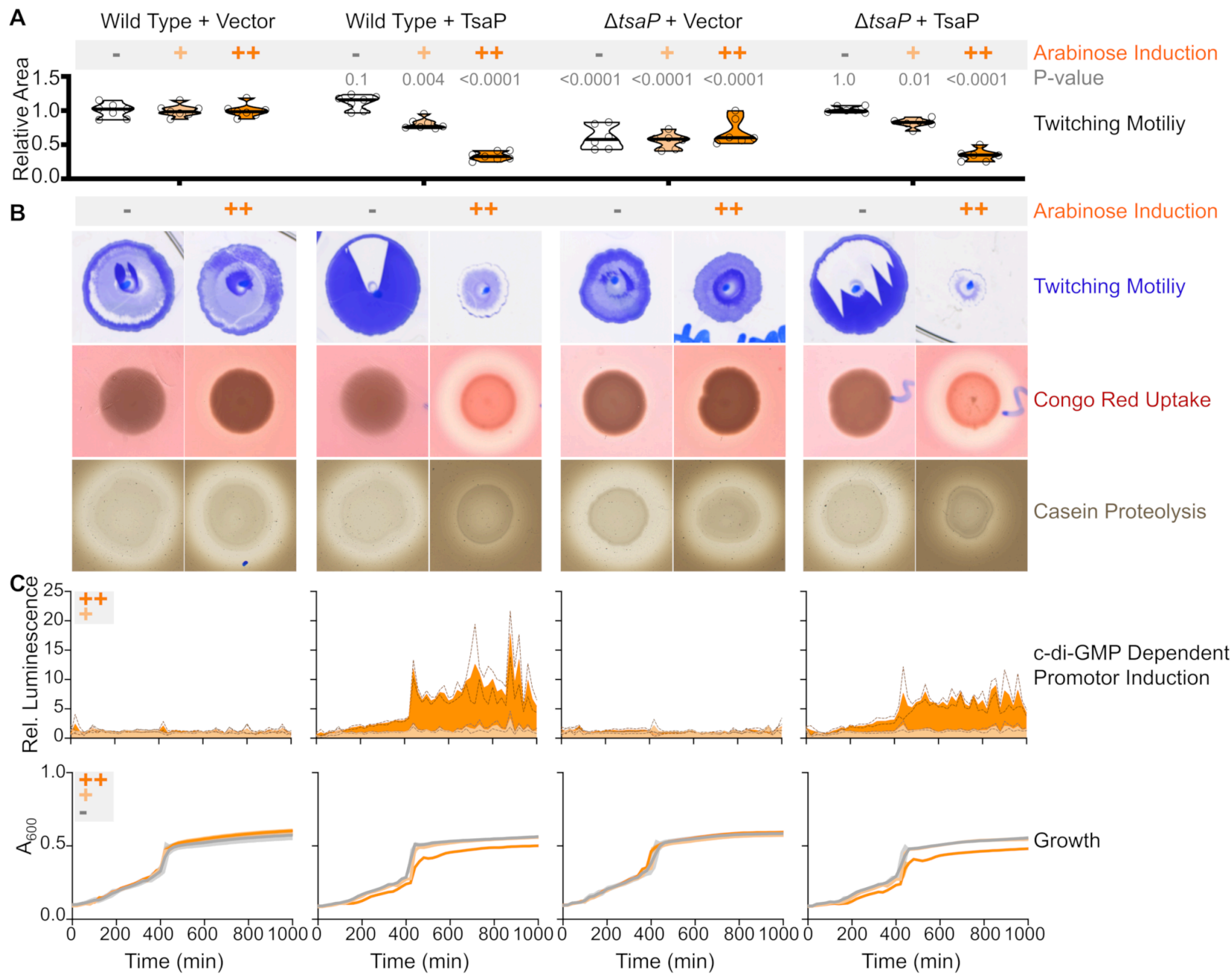

\section{D}
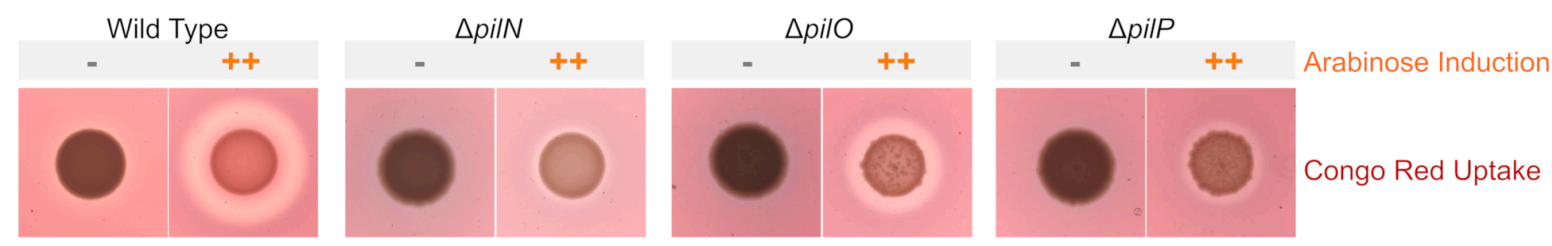

$\triangle p i l Q$

$\triangle$ pilF
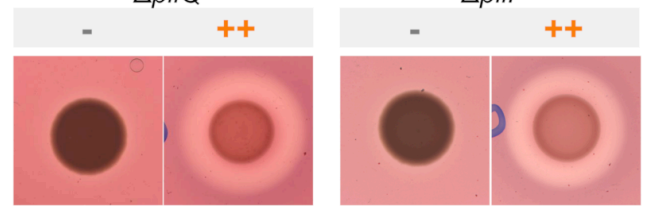

$\triangle$ pilA

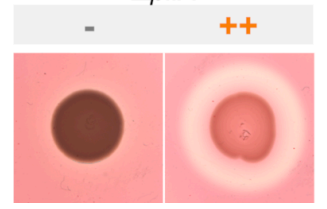

$\triangle p i l Y 1$

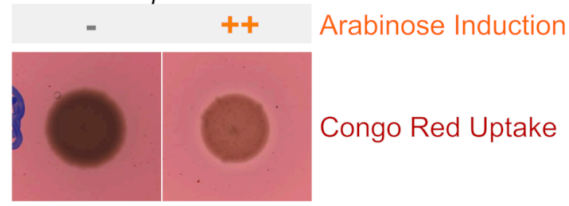

